\title{
Joint state-parameter estimation for tumor growth model
}

\author{
Annabelle Collin ${ }^{1}$, Thibaut Kritter ${ }^{1}$, Clair Poignard ${ }^{1}$, and Olivier Saut ${ }^{1}$ \\ ${ }^{1}$ Inria Bordeaux Sud-Ouest, Université de Bordeaux (IMB), CNRS and Bordeaux INP, \\ UMR 5251, F-33400, Talence, France
}

\begin{abstract}
We present a shape-oriented data assimilation strategy suitable for front-tracking tumor growth problems. A general hyperbolic/elliptic tumor growth model is presented as well as the available observations corresponding to the location of the tumor front over time extracted from medical imaging as MRI or CT scans. We provide sufficient conditions allowing to design a state observer by proving the convergence of the observer model to the target solution, for exact parameters. In particular, the similarity measure chosen to compare observations and simulation of tumor contour is presented. A specific joint stateparameter correction with a Luenberger observer correcting the state and a reduced-order Kalman filter correcting the parameters is introduced and studied. We then illustrate and assess our proposed observer method with synthetic problems. Our numerical trials show that state estimation is very effective with the proposed Luenberger observer, but specific strategies are needed to accurately perform parameter estimation in a clinical context. We then propose strategies to deal with the fact that data is very sparse in time and that the initial distribution of the proliferation rate is unknown. The results on synthetic data are very promising and work is ongoing to apply our strategy on clinical cases.
\end{abstract}

Keywords: Tumor growth, Data assimilation, Image processing, Front propagation

\section{Introduction}

During the last decade, the importance of mathematics in cancer biology has dramatically increased. Interdisciplinary interactions between mathematicians and biologists are now common and many different models have been derived to describe many different cancers. Yet concrete clinical applications of applied mathematics are rare. One reason is probably that most theoretical models are built from biological or pre-clinical observations and are difficult to apply in a clinical context. In particular, there are few models able to compute relevant personalized predictions for clinicians. Indeed, the models involve parameters that highly depend on the patient and on the targeted cancer, making the model calibration from clinical data very crucial, but still under investigated. Some recent preliminary studies of our team have investigated the calibration by fitting the volume of the tumor, without accounting for the tumor shape, providing thus unrealistic simulations [7]. To the best of our knowledge the estimation of the tumor shape over time via a sequential approach has not been addressed before. One can found simple trial-error strategies, focusing on the parameter estimation and not on the state - shape - estimation [18, 19], variational approaches [17] or through approximate analytical solutions with a reduced model [20]. The goal of this paper is to present joint state-parameter estimation strategy for a hyperbolic-elliptic tumor growth model, to combine imaging data (MRI or CT scans) with the model to improve the predictive potential of the simulation and to quantify the norm of the estimation. Roughly speaking, the aim is to combine imaging data of the tumor - that is the tumor shape - at different time points to improve the model calibration and thus the predictive ability of the model.

Front-tracking problems appear in the study of moving interfaces at the macroscopic level. They arise in many applications such as fire propagation [31], weather forecasting [2], 
cardiac electrophysiology [9], oil spill [24], and tumor growth [26]. Tracking the contour and the motion of such objects is challenging because of motion irregularities and topological changes. A model cannot perfectly describe the evolution because of physical or biological simplifications. Moreover, initial front position is subject to measurement uncertainties, and model parameters are usually difficult to identify. Data assimilation [30] aims at solving these issues and accounting for the uncertainties. The idea is to integrate observations into the model in order to correct contour errors. Front positions are then estimated more precisely and predictions are improved. These methods focus on correcting the state and/or the parameters of the model. Evaluating a correction requires to know how to compare the simulated state and the observations contours. The choice of contour discrepancy is thus fundamental in data assimilation.

Our goal here lies in the modeling of tumor growth. In previous works of our research team, the evolution of the tumor shape has been estimated by fitting a growth model $[7,10]$ through a reduced-order model corresponding to the tumoral volume evolution. This strategy may be used when tumor shapes are almost spherical but the estimation of the tumor shape on non-trivial shape was not addressed. The idea here is to complete the tumor growth model with data assimilation strategy in order to predict tumor shape evolution. More precisely, observations are used to correct the state and the model parameters. This estimation provides crucial information in the case of brain or pulmonary tumors for instance, since the tumor may affect vital functions or structures of the ill organ.

The paper is outlined as follows. In Section 2, the general hyperbolic/elliptic tumor growth model is presented as well as the available observations (tumor fronts extracted from MRI or CT scans). In addition to the cell proliferation, our model makes it possible to account for the influence of the tumor vascularization or the treatment in the same mathematical framework. We then present the principles of data assimilation strategy applied to our purpose. Section 3, is devoted to the analysis of a state observer. We provide sufficient conditions allowing to design a state observer by proving the convergence of the observer model to the target solution, for exact parameters. The proof relies on the hyperbolic properties of the tumor model, combined with Gagliardo-Nirenberg estimates in Sobolev spaces. A joint state-parameter correction is then developed in Section 4, as initiated in [29], with a Luenberger observer correcting the state and a reduced-order Kalman filter [28, 27] correcting the parameters. In particular, we introduce the similarity measure chosen to compare observations and simulation of tumor contour. In Section 5, the presentation of the numerical approximation and the numerical validation of the state observer are given. We eventually validate our strategy on synthetic data. This paper lays the foundations for data assimilation strategy - based on sequential approaches - in mathematical oncology.

\section{Problem setting}

\subsection{Tumor growth model and observations}

In this paper a generic hyperbolic/elliptic tumor growth model is studied. Let $\mathcal{B}$ be a bounded and smooth domain of $\mathbb{R}^{d}, d=2,3$. Two different cell types are considered, the tumor cells denoted by $P$ and the healthy cells of the host tissue $S$. The saturation assumption is chosen to deduce $S$ from $P$ thanks to $S+P=1$. Two functionals denoted by $\mathcal{G}^{i}, i=0,1$ describe respectively the tumor evolution $\left(\mathcal{G}_{0}\right)$ and the induced pressure due to tumor expansion $\left(\mathcal{G}_{1}\right)$. We denote by $v$ the velocity field that describes the motion of tumor cells and by $\pi$ the pressure. The generic non linear model of tumor growth reads as follows:

$$
\left\{\begin{array}{rlrl}
\partial_{t} P+\nabla \cdot(v P) & =\mathcal{G}^{0}(t, P), & \mathcal{B}, \\
\nabla \cdot v & =\mathcal{G}^{1}(t, P), & \mathcal{B} \\
v & =-\nabla \pi, & \mathcal{B} \\
\left.\pi\right|_{\partial \mathcal{B}} & =0, & & \partial \mathcal{B} \\
P(0, \cdot) & =P_{\bullet}, & \mathcal{B}
\end{array}\right.
$$


The homogeneous Dirichlet boundary condition (1d) accounts for the fact that the pressure far from the tumor is not affected by the growth. This hypothesis is validated only if $\mathcal{B}$ is large enough. This has to be numerically verified. When there exists a mechanical constraint to the growth, for example the skull, homogeneous Neumann conditions are considered at the boundary of the constrained domain. It is worth noting that other tumor rheologies than Darcy's law might be used to account for instance for the surface tension or other specific behavior of the tumor [4]. However the Darcy law has been proven to provide relevant results regarding the clinical applications, see for instance $[3,8,23]$ and references therein.

For the sake of simplicity, we restrict our study to growing tumors -that is $\mathcal{G}^{1}$ is a positive functional- meaning that the velocity is outwardly directed. Therefore no boundary conditions are necessary on the cell densities $P$ and $S$. We are confident that adding an effect of a treatment can be easily assessed by adding a boundary condition on $P$ and by adapting the boundary condition on $\pi$.

Remark 1 (The particular case of tumor growth influence on vascularization). The hypotheses on $\mathcal{G}^{i}, i=0,1$ is specified later on, however the simplest case of a growing tumor is obtained with $\mathcal{G}^{0}(t, P)=\mathcal{G}^{1}(t, P)=\alpha P$, where $\alpha$ is the proliferating rate of tumor cells. More interestingly, if the tumor cells impacts on the vascularization, one can consider

$$
\mathcal{G}^{0}(t, P)=\mathcal{G}^{1}(t, P)=M_{0}(\cdot) P(t, \cdot) e^{-\alpha \int_{0}^{t} P(s, \cdot) d s} .
$$

The rationale of this choice of functionals is the following. The cell proliferation generates an inner gradient pressure that moves both cancer and healthy cells. The local nutrient supply is accounted for the proliferation rate $e^{-\alpha \int_{0}^{t} P(s, \cdot) d s}$. This rate is supposed to be degraded by the cancer cell at the consumption rate $\alpha>0$. The model also accounts for a very fast growth, when $\alpha<0$. Biologically, $\alpha<0$ means that the tumor is able to vascularize itself, which corresponds to angiogenesis phenomenon. The spatial parameter $M_{0}$ can be seen at the initial proliferating rate. This case is detailed in Section 4.

The generic model (1) cannot be straightforwardly used to provide clinically relevant results. Indeed, the model is rather simplistic and the tumor segmentation at the initial time generates geometric uncertainties and the involved parameters cannot be measured. The uncertainties on data are linked to the parameters of the functionals $\mathcal{G}_{i}, i=0,1$, that is for instance the consumption and proliferating rates $\alpha$ and $M_{0}$ (which may be spatially distributed) in the case of (2). Regarding the initial condition on $P$, note that it can be directly computed from the segmentation of the first available clinical imaging data, however uncertainties due to the segmentation imply uncertainties on $P_{\bullet}$.

Fortunately, additional imaging information is acquired during the patient follow-up. Indeed, the patient follow-up consists of a time series of medical imaging data (MRI or CT scans for example) performed at a regular frequency (which can be several months). Here we investigate how this new data may be used to circumvent the uncertainties associated with the dynamical model. Concerning measurement uncertainties - see [11] for a study of uncertainties for manual 2D segmentations for lung or liver metastasis - they can be directly considered in sequential data assimilation as we will see in what follows.

Using, for example, a semi-automatic segmentation approach, a mask of the tumor can be extracted at each acquisition time. Theoretically speaking, working with a discrete sequence of images is a major issue in data assimilation. That is why, we assume in the first place (for Section 3) that measurements can be considered as a time-continuous sequence of tumor masks, namely, maps taking essentially two different values inside and outside the tumor region, up to some perturbations and regularization across the front (in order to have smooth enough data). In the numerical resolution section, we see how to reconstruct a continuous sequence from a discrete one. 


\subsection{Principle of sequential data assimilation}

The aim of sequential data assimilation approach is to correct the model dynamic using observation data. Let us consider the general following system - called the target model

$$
\left\{\begin{aligned}
\partial_{t} y(t, x) & =\mathcal{M}(t, y(t, x), \theta) \\
y(0, x) & =y_{\bullet}(x)+\zeta_{y}(x) \\
\theta & =\theta_{\bullet}+\zeta_{\theta}
\end{aligned}\right.
$$

with $y$ the state of the system, $\mathcal{M}$ the dynamics and $\theta$ is a vector concatenating all the parameters of the model (assumed to be constant in time). We consider that the initial condition $y(0, x)$ (resp. the vector of parameters $\theta$ ) is decomposed into two parts $y_{\bullet}(x)+\zeta_{y}(x)$ (resp. $\theta_{\bullet}+\zeta_{\theta}$ ), where $y_{\bullet}\left(\right.$ resp. $\left.\theta_{\bullet}\right)$ is the a-priori part and $\zeta_{y}$ (resp. $\zeta_{\theta}$ ) the uncertainty. We denote by $z:(t, x) \mapsto z(t, x)$ the available observation. We define by $\mathcal{D}(z, y)$ a discrepancy operator which measures the error between the state $y$ and the observations $z$.

Sequential approaches correct the dynamics by a feedback based on the discrepancy combining data and the model state. This modified model is called the observer model. In the present article, we propose a joint state and parameter observer formulation following [29]. A Luenberger observer-namely an appropriate penalization term involving the discrepancy with the measurements- corrects the state [25], and a reduced-order optimal Kalman-based filter [28] is restricted to the parametric space reducing the computational cost. The dynamics of the joint state and parameter observer can be written as

$$
\left\{\begin{aligned}
\partial_{t} \hat{y}(t, x) & =\mathcal{M}(t, \hat{y}(t, x), \theta)+\underline{\mathcal{G}}_{y}(\mathcal{D}(z(t, x), \hat{y}(t, x))) \\
\dot{\hat{\theta}}(t) & =\mathcal{G}_{\theta}(\mathcal{D}(z(t, x), \hat{y}(t, x))) \\
\hat{y}(0, x) & =y_{\bullet}(x) \\
\theta(0) & =\theta_{\bullet}
\end{aligned}\right.
$$

where $\underline{\mathcal{G}}_{y}$ and $\mathcal{G}_{\theta}$ are gain operators, also called filters, which have to be chosen together with an appropriate Banach space $\mathcal{X}$ embedded with a well-chosen norm space $\|\cdot\| \mathcal{X}$ so that

$$
\|\hat{y}(t, \cdot)-y(t, \cdot)\|_{\mathcal{X}} \longrightarrow_{t \rightarrow \infty} 0, \quad \text { and } \quad \hat{\theta}(t) \longrightarrow_{t \rightarrow \infty} \theta
$$

where $y$ corresponds to the solution of Equation (3) and is called the target solution. It is worth noting that in [9], which dealt with reaction-diffusion system, the space $\mathcal{X}$ is $L^{2}$ with its usual norm.

The next section focuses on defining a discrepancy and a state observer

$$
\left\{\begin{aligned}
\partial_{t} \hat{y}(t, x) & =\mathcal{M}(t, \hat{y}(t, x), \theta)+\mathcal{G}_{y}(\mathcal{D}(z(t, x), \hat{y}(t, x))) \\
\hat{y}(0, x) & =y_{\bullet}(x) \\
\theta(0) & =\theta_{\bullet}+\zeta_{\theta}
\end{aligned}\right.
$$

allowing the convergence $\|\hat{y}(t, \cdot)-y(t, \cdot)\|_{\mathcal{X}} \longrightarrow_{t \rightarrow \infty} 0$, when the uncertainties are reduced to the initial conditions (i.e. parameters are fixed to their true values). In order to have a consistent observer, we expect the correction term to vanish when $\hat{y}=y$, namely, we should have

$$
\mathcal{G}_{y}(\mathcal{D}(z(t, x), y(t, x)))=0 .
$$

Once well defined discrepancy and state filter have been chosen and validated, the joint state and parameter observer derive from the previous works of Chapelle, Moireau et al. [29, 28].

\section{Analysis of the state observer}

To design a state observer, we assume that the uncertainties are reduced to the initial condition $P_{\bullet}$. Throughout the paper, for the sake of simplicity we assume that the domain 
$\mathcal{B}$ is a smooth domain of $\mathbb{R}^{3}$. This section is decomposed into two parts. First, we study the well-posedness of the model (1) in appropriate Sobolev spaces for small time, see Proposition 1 and the boundedness of the solution, see Proposition 2. Then, we analyze a linearized version of a state observer.

\subsection{Well-posedness in appropriate Sobolev spaces for small time}

We assume that the functionals $\mathcal{G}^{i}, i=0,1$ are defined from $\mathbb{R}^{+} \times L^{\infty}\left(0, T ; H^{s}(\mathcal{B})\right)$ into $L^{\infty}\left(0, T ; H^{s}(\mathcal{B})\right)$. In addition, they satisfy the following Hypothesis 1 :

Hypothesis 1 (Hypothesis on the functional mappings $\mathcal{G}^{i}, i=0,1$ ).

- For $i=0,1, \mathcal{G}^{i}$ is a uniformly bounded and smooth functional which maps $\mathbb{R}^{+} \times$ $L^{\infty}\left(0, T ; H^{s}(\mathcal{B})\right)$ into $L^{\infty}\left(0, T ; H^{s}(\mathcal{B})\right)$ for any $s \geq d / 2$.

- For any $P \in L^{\infty}\left(0, T ; H^{s}(\mathcal{B})\right)$, the function $g_{P}$ is defined on $\mathbb{R}^{+} \times \mathcal{B}$ by

$$
g_{P}(t, x)=\mathcal{G}^{0}(t, P(t, x))-P \mathcal{G}^{1}(t, P(t, x)) .
$$

The function $g_{P}$ is such that for any $P \in L^{\infty}\left(0, T ; H^{s}(\mathcal{B})\right), g_{P}$ belongs to $L^{\infty}\left(0, T ; H^{s}(\mathcal{B})\right)$. Moreover if $P(t, \cdot)$ compactly supported in $\mathcal{B}$, then $g_{P}(t, \cdot)$ is compactly supported in $\mathcal{B}$.

The first objective of this subsection is to prove the following proposition.

Proposition 1. Assume that $P_{\bullet} \in H^{s}(\mathcal{B})$ such that the support of $P_{\bullet}$ is compactly embedded in $\mathcal{B}$. Then there exists $T$ small enough such that Problem (1) admits a unique solution in $L^{\infty}\left(0, T ; H^{s}(\mathcal{B})\right)$ whose support is compactly embedded in $\mathcal{B}$.

The proof of the proposition is based upon the following lemmas.

Lemma 1 (Elliptic regularity). Denote by $\Psi$ the functional defined by

$$
\Psi: \quad P \mapsto v, \text { where } v \text { is the solution to }(1 \mathrm{~b})-(1 \mathrm{c})-(1 \mathrm{~d}) .
$$

For any $T>0$, for any $s \geq 0, \Psi$ is a Lipschitz mapping from the space $L^{p}\left(0, T ; H^{s}(\mathcal{B})\right)$ in the space $L^{p}\left(0, T ; H^{s+1}(\mathcal{B})\right)$, for any $P \in[1,+\infty]$. More precisely, there exists $C$ such that

$$
\begin{aligned}
& \|\Psi(P)\|_{L^{p}\left(0, T ; H^{s+1}(\mathcal{B})\right)} \leq C\|P\|_{L^{p}\left(0, T ; H^{s}(\mathcal{B})\right)}, \quad \forall P \in L^{p}\left(0, T ; H^{s}(\mathcal{B})\right) \\
& \|\Psi(P)-\Psi(\underline{P})\|_{L^{p}\left(0, T ; H^{s+1}(\mathcal{B})\right)} \leq C\|P-\underline{P}\|_{L^{p}\left(0, T ; H^{s}(\mathcal{B})\right)}, \quad \forall P, \underline{P} \in L^{p}\left(0, T ; H^{s}(\mathcal{B})\right)
\end{aligned}
$$

The proof of this lemma is standardly based on energy estimates thanks to the fact that $\mathcal{G}^{1}$ is continuous in $t$ and Lipschitz in its second argument-more precisely, $\mathcal{G}^{1}$ is smooth in both arguments- from $\mathbb{R}^{+} \times L^{\infty}\left(0, T ; H^{s}(\mathcal{B})\right)$ into $L^{\infty}\left(0, T ; H^{s}(\mathcal{B})\right)$ by definition of $\mathcal{G}^{1}$.

Remark 2. Thanks to equality (1b), the transport equation (1a) on $P$ reads in the nonconservative form as

$$
\partial_{t} P+v \cdot \nabla P=\mathcal{G}^{0}(t, P)-P \mathcal{G}^{1}(t, P) .
$$

The above lemma shows that the velocity is bounded if $P$ is smooth enough. Combining this lemma with the assumption on $\mathcal{G}^{i}, i=0,1$ and the fact that the support of $P_{\bullet}$ is compactly embedded in $\mathcal{B}$ ensure that the cell density $P$ does not touch the boundary of $\mathcal{B}$ during its existence time.

The next lemma is crucial to ensure the stability in Sobolev spaces of high enough order.

Lemma 2. Let $s>d / 2$, and $v \in H^{s+1}(\mathcal{B})$. For any $m \in[0, s]$, for any $u \in H^{m}(\mathcal{B})$ such that the $\operatorname{supp}(u)$ is compactly embedded in $\mathcal{B}$, then the following estimate holds:

$$
\left|\int_{\mathcal{B}} \partial_{x}^{m} \nabla \cdot(v u) \partial_{x}^{m} u d x\right| \leq C\|v\|_{H^{s+1}(\mathcal{B})}\|u\|_{H^{m}(\mathcal{B})}^{2}
$$


The proof of the above lemma relies on the appropriate use of Gagliardo-Nirenberg estimates [1], and the fact that for $m>d / 2, H^{m}(\mathcal{B})$ is continuously embedded in $L^{\infty}(\mathcal{B})$ and the Sobolev spaces $H^{m}(\mathcal{B})$ are algebra, meaning that the product of Sobolev functions of order $m$ is still a Sobolev function of order $m$. For the sake of conciseness it is left to the reader.

Lemma 3. Let $P_{\bullet} \in H^{s}(\mathcal{B})$ such that the support of $P_{\bullet}$ is compactly embedded in $\mathcal{B}$. Denote by $\Phi$ the functional defined by

$$
\Phi:\left\{\begin{aligned}
L^{2}\left(0, T ; H^{s+1}\right) & \rightarrow L^{\infty}\left(0, T ; H^{s}\right) \\
v & \mapsto P
\end{aligned}\right.
$$

where $P$ is the solution to $(1 \mathrm{a})-(1 \mathrm{e})$.

For $s>5 / 2$, there exists $C$ such that

$$
\begin{array}{r}
\|\Phi(v)\|_{L^{\infty}\left(0, T ; H^{s}(\mathcal{B})\right)}^{2} \leq C\|P \bullet\|_{H^{s}(\mathcal{B})}^{2} e^{C \sqrt{T}\left(1+\|v\|_{L^{2}\left(0, T ; H^{s+1}(\mathcal{B})\right)}\right)}, \\
\|\Phi(v)-\Phi(\tilde{v})\|_{L^{\infty}\left(0, T ; H^{s}(\mathcal{B})\right)}^{2} \leq C\|P \bullet\|_{H^{s}(\mathcal{B})}^{2}\|v-\tilde{v}\|_{L^{2}\left(0, T ; H^{s+1}(\mathcal{B})\right)}^{2} e^{C \sqrt{T}\left(1+\|v\|_{L^{2}\left(0, T ; H^{s+1}(\mathcal{B})\right)}\right)} .
\end{array}
$$

Proof. We prove the result for $m \in \mathbb{N} \backslash 0,1,2$. The result is then extended to $s>5 / 2$ by interpolation and continuous Sobolev embedding in $L^{\infty}$.

As standard in the analysis of nonlinear transport equations [1], we apply the derivative operator $\partial_{x}^{n}$ to equation (1a), for $n=0, \cdots, m$, with $m \leq s$. Multiplying the resulting equality by $\partial_{x}^{n} P$ and integrating with respect to $x$ to infer

$$
\frac{1}{2} \frac{d}{d t}\left\|\partial_{x}^{n} P(t, \cdot)\right\|_{L^{2}(\mathcal{B})}^{2}+\int_{\mathcal{B}} \partial_{x}^{n} \nabla \cdot(v P) \partial_{x}^{n} P d x=\int_{\mathcal{B}} \partial_{x}^{n}\left(\mathcal{G}^{0}(t, P)\right) \partial_{x}^{n} P d x
$$

Thanks to the definition of $\mathcal{G}^{0}$ and thanks to Lemma 2, by summing the above equality from $n=0, \cdots, m$ we infer that there exists a constant $C$ such that

$$
\frac{d}{d t}\|P(t, \cdot)\|_{H^{m}(\mathcal{B})}^{2} \leq C\left(1+\|v(t, \cdot)\|_{H^{s+1}}\right)\|P(t, \cdot)\|_{H^{m}(\mathcal{B})}^{2}
$$

To prove that $\Phi$ is a contraction, let $(v, \tilde{v}) \in\left(L^{2}\left(0, T ; H^{s+1}(\mathcal{B})\right)\right)^{2}$, the function $Q=P-\tilde{P}$ satisfies

$$
\left\{\begin{array}{l}
\partial_{t} Q+\nabla \cdot(v Q)=\mathcal{G}^{0}(t, P)-\mathcal{G}^{0}(t, \tilde{P})-\nabla \cdot((v-\tilde{v}) \tilde{P}), \\
\left.Q\right|_{t=0}=0
\end{array}\right.
$$

Similar reasoning on $\|Q(t, \cdot)\|_{H^{m}}$ shows then that

$\frac{d}{d t}\|Q(t, \cdot)\|_{H^{m}}^{2} \leq C\left(\|P(t, \cdot)\|_{H^{m}}^{2}+\|\tilde{P}(t, \cdot)\|_{H^{m}}^{2}\right)\left\{\left(1+\|v(t, \cdot)\|_{H^{s+1}}\right)\|Q(t, \cdot)\|_{H^{m}}^{2}+\|v-\tilde{v}\|_{H^{s+1}}^{2}\right\}$.

Gronwall's lemma leads to the estimates (11).

We are now ready to prove Proposition 1.

Proof of Proposition 1. To prove the local well-posedness, let $\mathcal{E}_{R}$ the subset of $L^{\infty}\left(0, T ; H^{s}\right)$ be defined as

$$
\mathcal{E}_{R}:=\left\{p \in L^{\infty}\left(0, T ; H^{s}\right):\|p\|_{L^{\infty}\left(0, T ; H^{s}\right)} \leq R, \quad \text { and } \quad \operatorname{supp}(p) \Subset \mathcal{B}\right\}
$$

be endowed with the usual norm of the Banach space $L^{2}\left(0, T ; H^{s-1}\right)$. Since the closed unit ball in $L^{2}\left(0, T ; H^{s-1}\right)$ is compact in the weak topology and the closed unit balls in $L^{\infty}\left(0, T ; H^{s-1}\right)$ and in $L^{\infty}\left(0, T ; H^{s}\right)$ are compact in the weak ${ }^{*}$ topology [5], we get that $\mathcal{E}_{R}$ is a closed subspace of $L^{2}\left(0, T ; H^{s-1}\right)$. Then Lemmas $3-1$ imply that there exist a $T$ small enough such that the operator $\Lambda=(\Phi \circ \Psi)^{2}$, is a contraction in $\mathcal{E}_{R}$. Existence and uniqueness of the fixed point of $\Lambda$ thus follows, which leads to the existence and uniqueness of the fixed point $\Phi \circ \Psi$ in $L^{2}\left(0, T ; H^{s-1}\right)$ and thus by stability in $L^{\infty}\left(0, T ; H^{s}\right)$. 
Proposition 2. Let $s \geq 5 / 2$ and assume that $P_{\bullet} \in H^{s}(\mathcal{B})$ such that the support of $P_{\bullet}$ is compactly embedded in $\overline{\mathcal{B}}$, and assume in addition that

$$
0 \leq P_{\bullet} \leq 1, \quad \text { a.e in } \mathcal{B}
$$

Let $\mathcal{L}^{0}$ satisfy Hypothesis 1 , and set

$$
\forall u \in L^{\infty}\left(0, T ; H^{s}(\mathcal{B})\right), \quad \mathcal{G}_{0}(t, u)=\mathcal{G}_{1}(t, u)=u \mathcal{L}^{0}(t, u) .
$$

For $i=0,1$, it is obvious that $\mathcal{G}_{i}$ satisfies Hypothesis 1 , and let $T$ be such that the unique solution $P$ of $(1)$ exists in $L^{\infty}\left(0, T ; H^{s}(\mathcal{B})\right)$, and such that the support of $P$ is compactly embedded in $\mathcal{B}$ then for any $t \in(0, T)$,

$$
0 \leq P(t, x) \leq 1
$$

Proof. Let $P^{-}$be the negative part of $P$. That is $P=P^{+}-P^{-}$. Multiplying the transport equation (1a) by $P^{-}$, and integrating over $\mathcal{B}$ leads to

$$
-\frac{1}{2} \frac{d}{d t}\left\|P^{-}(t, \cdot)\right\|_{L^{2}(\mathcal{B})}^{2}-\frac{1}{2} \int_{\mathcal{B}} v \nabla\left(\left(P^{-}\right)^{2}\right) d x=-\int_{\mathcal{B}} \mathcal{L}^{0}(t, P)\left(P^{-}\right)^{2}\left(1+P^{-}\right) d x .
$$

Then using the fact that $P$ vanishes in a neighborhood of $\partial \mathcal{B}$ and using (1b) leads to

$$
\frac{d}{d t}\left\|P^{-}(t, \cdot)\right\|_{L^{2}(\mathcal{B})}^{2}=\int_{\mathcal{B}} \mathcal{L}^{0}(t, P)\left(P^{-}\right)^{2}\left(2+P^{-}\right) d x .
$$

By Proposition 1, there exists $C>0$ such that

$$
\|P\|_{L^{\infty}\left(0, T ; H^{s}(\mathcal{B})\right)} \leq C,
$$

thus there exists a constant still denoted by $C$ such that

$$
\frac{d}{d t}\left\|P^{-}(t, \cdot)\right\|_{L^{2}(\mathcal{B})}^{2} \leq C \int_{\mathcal{B}}\left(P^{-}\right)^{2} d x
$$

from which we infer that since $P_{\bullet}^{-}=0$, then for any $t \in(0, T), P^{-}(t, \cdot)=0$, and thus $P \geq 0$. Similar reasoning on $Q=1-P$ implies that $P \leq 1$.

\subsection{Analysis of the linearized state observer}

This section is devoted to the analysis of the convergence of the state observer towards the target. Let $\mathcal{L}^{0}$ such that $\mathcal{G}^{0}=\mathcal{G}^{1}=\mathcal{L}^{0} P$ satisfy Hypothesis 1 , we denote by $P$ the solution to the following tumor growth model:

$$
\left\{\begin{aligned}
\partial_{t} P+\nabla \cdot(v P) & =\mathcal{L}^{0}(t, P) P, & \mathcal{B} \\
P(0, \cdot) & =P_{\bullet}+\zeta_{P}(x), & \mathcal{B} \\
\nabla \cdot v & =\mathcal{L}^{0}(t, P) P, & \mathcal{B} \\
v & =-\nabla \pi, & \mathcal{B} \\
\left.\pi\right|_{\partial \mathcal{B}} & =0, & \partial \mathcal{B}
\end{aligned}\right.
$$

where $\zeta_{P}$ is the uncertainty on the initial condition. We want to study the following state observer model:

$$
\left\{\begin{aligned}
\partial_{t} \hat{P}+\nabla \cdot(\hat{v} \hat{P}) & =\mathcal{L}^{0}(t, \hat{P}) \hat{P}-\lambda f(z, \hat{P}), & \mathcal{B} \\
\hat{P}(0, \cdot) & =P_{\bullet}, & \mathcal{B} \\
\nabla \cdot \hat{v} & =\mathcal{L}^{0}(t, \hat{P}) \hat{P}, & \mathcal{B} \\
\hat{v} & =-\nabla \hat{\pi}, & \mathcal{B} \\
\left.\hat{\pi}\right|_{\partial \mathcal{B}} & =0, & \partial \mathcal{B}
\end{aligned}\right.
$$


where following the notation of System (5) the state filter is assumed to be written as

$$
\mathcal{G}_{P}(\mathcal{D}(z(t, x), \hat{P}(t, x)))=-\lambda f(z, \hat{P})
$$

where $\lambda>0$ is called the gain parameter.

Throughout the paper, we consider that $f$ designs a consistent observer, and that it provides physically realistic solutions.

Hypothesis 2. We assume that $f$ satisfies the following assumptions:

Consistent observer If $P$ is the solution of the target system (12), then $f(z, P)=0$, hysically realistic solutions For any $u \in L^{\infty}\left(0, T ; H^{s}\right)$ for s large enough,

$$
\left\{\begin{array}{l}
\text { If } u \leq 0 \text { then } f(z, u) \leq 0, \\
\text { If } u \geq 1 \text { then } f(z, u) \geq 0 .
\end{array}\right.
$$

Proposition 3. Assume that $P_{\bullet}$ and $\zeta_{P}$ are such that

$$
0 \leq P_{\bullet} \leq 1, \quad 0 \leq P_{\bullet}+\zeta_{P} \leq 1, \quad \text { in } \mathcal{B} .
$$

Assume in addition that $\mathcal{L}^{0}$ is such that the solution to Problem (12) exists in $L^{\infty}\left(0,+\infty ; H^{s}\right)$. Finally assume that $f$ satisfies Hypothesis 2 and is such that for any $\lambda>0$, the solution to Problem (15) exists in $L^{\infty}\left(0,+\infty ; H^{s}\right)$. Then

$$
0 \leq \hat{P} \leq 1, \quad \text { a.e. in }(0,+\infty) \times \mathcal{B} .
$$

The proof is obvious thanks to assumption (14).

In order to investigate the convergence of the state observer towards the target solution, we only consider the linearization of the observer term $f$

$$
f(z, \hat{P}) \approx-d f(z, P)(P-\hat{P}) .
$$

This approximation is valid when the difference between $\hat{P}$ and $P$ is assumed to be small (i.e. the uncertainty of the initial condition is assumed to be reasonable).

We denote by $\hat{P}$ the solution to the following linearized state observer model in the sense of distribution:

$$
\left\{\begin{aligned}
\partial_{t} \hat{P}+\nabla \cdot(\hat{v} \hat{P}) & =\mathcal{L}^{0}(t, \hat{P}) \hat{P}+\lambda d f(z, P)(P-\hat{P}), & \mathcal{B} \\
\hat{P}(0, \cdot) & =P_{\bullet}, & \mathcal{B} \\
\nabla \cdot \hat{v} & =\mathcal{L}^{0}(t, \hat{P}) \hat{P}, & \mathcal{B} \\
\hat{v} & =-\nabla \hat{\pi}, & \mathcal{B} \\
\left.\hat{\pi}\right|_{\partial \mathcal{B}} & =0, & \partial \mathcal{B}
\end{aligned}\right.
$$

According to Proposition 1 there exists $T$ small enough such that the unique solution to Problem (12) exists and belongs to $L^{\infty}\left(0, T ; H^{s}\right), s>5 / 2$. In addition if $d f(z, P)$ maps $L^{\infty}\left(0, T ; H^{s}\right)$ into itself for $s>5 / 2$, thus for any $\lambda>0$, there exists a unique solution to Problem (15) in $L^{\infty}\left(0, T^{\lambda} ; H^{s}\right), s>5 / 2$ with $T^{\lambda}<T$. However we are not interested in the condition to ensure global existence. We assume in the following proposition that $\mathcal{L}^{0}$ and $f$ are well-chosen to ensure the global existence of the solution to the two problems (12) and (15).

Proposition 4. Assume also that $P_{\bullet}$ and $\zeta_{P}$ are such that

$$
0 \leq P_{\bullet} \leq 1, \quad 0 \leq P_{\bullet}+\zeta_{P} \leq 1, \quad \text { in } \mathcal{B} .
$$

Assume in addition that $\mathcal{L}^{0}$ is such that the solution to Problem (12) exists in $L^{\infty}\left(0,+\infty ; H^{s}\right)$ and that $f$ is such that for any $\lambda>0$, the solution to Problem (15) exists in $L^{\infty}\left(0,+\infty ; H^{s}\right)$. 
Remark 3. The simplest case of $\mathcal{L}^{0}$ for which global existence of System (12) is given by

$$
\mathcal{L}^{0}(t, P)=M_{0}(x) e^{-\alpha t},
$$

where $M_{0}$ is smooth enough with compact support strictly embedded in $\mathcal{B}$, and $M_{0}, P_{0}$ and $\alpha$ are such that $P$ never touches $\partial \mathcal{B}$. This case corresponds to the fact the the tumor degrades the tissue vascularization everywhere in $\mathcal{B}$ with the same rate $\alpha$. In Remark $1, \mathcal{L}^{0}$ is given by

$$
\mathcal{L}^{0}(t, P)=M_{0}(x) e^{-\alpha \int_{0}^{t} P(s, x) d s},
$$

for which global existence also occurs for $M_{0}$ smooth with compact support strictly embedded in $\mathcal{B}$, and with $M_{0}, P_{0}$ and $\alpha$ are such that $P$ never touches $\partial \mathcal{B}$. This case corresponds to the fact the the tumor degrades the tissue vascularization only inside the tumor.

More generally, global well-posedness of System (12) and (13) occurs as soon as the terms $\mathcal{L}^{0}$ and $f$ map continuously $L^{\infty}\left(0,+\infty ; H^{s}\right)$ into itself and that at any time their support is compactly strictly embedded in $\mathcal{B}$ to avoid a loss of regularity near the boundary of $\mathcal{B}$.

- If there exist $\alpha>0$ and $\beta>0$ such that

$$
\int_{\mathcal{B}} d f(z, P)(u) u d x \geq \alpha\|u\|_{L^{2}(\mathcal{B})}^{2}-\beta, \quad \forall u \in L^{\infty}\left(0,+\infty ; H^{s}\right),
$$

then there exists $c>0$ such that for any $t>0$, for any $\lambda>0$,

$$
\|(\hat{P}-P)(t)\|_{L^{2}(\mathcal{B})}^{2} \leq\|(\hat{P}-P)(0)\|_{L^{2}(\mathcal{B})}^{2} e^{-(\lambda \alpha-c) t}+\frac{\lambda \beta\left(1-e^{-(\lambda \alpha-c) t}\right)}{\lambda \alpha-c} .
$$

In particular if $\beta=0$, for any $\lambda>c / \alpha, t \mapsto\|(\hat{P}-P)(t)\|_{L^{2}(\mathcal{B})}$ decreases exponentially towards 0.

- If there exists $\alpha>0$ and $p \geq 1$ such that

$$
\int_{\mathcal{B}} d f(z, P)(u) u d x \geq \alpha\|u\|_{L^{p}(\mathcal{B})}, \quad \forall u \in L^{\infty}\left(0,+\infty ; H^{s}\right),
$$

there exists $\lambda_{0}$ such that for any $\lambda>\lambda_{0, p}, t \mapsto\|(\hat{P}-P)(t)\|_{L^{2}(\mathcal{B})}^{2}$ decreases linearly towards 0. In addition, if $p=2$, then $t \mapsto\|(\hat{P}-P)(t)\|_{L^{2}(\mathcal{B})}$ decreases linearly towards 0 .

Proof. To prove the decay of $t \mapsto\|(\hat{P}-P)(t, \cdot)\|_{L^{2}(\mathcal{B})}^{2}$, consider the problem satisfied by $\tilde{P}:=P-\hat{P}$ and $\tilde{v}:=v-\hat{v}$ in $(0,+\infty) \times \mathcal{B})$

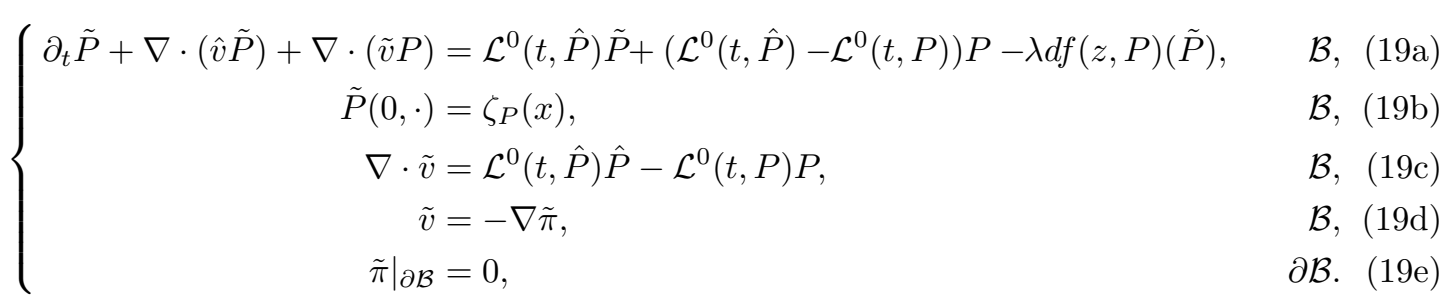

There exists a constant $C$ such that by definition of $\mathcal{L}^{0}$, and since $P \in L^{\infty}\left(0,+\infty, H^{s}(\mathcal{B})\right)$, with $s>5 / 2$, one has for any $t \in(0,+\infty)$ :

$$
\begin{aligned}
& \left\|\mathcal{L}^{0}(t, \hat{P})\right\|_{L^{\infty}(\mathcal{B})} \leq C, \\
& \left\|\mathcal{L}^{0}(t, \hat{P})-\mathcal{L}^{0}(t, P)\right\|_{L^{2}(\mathcal{B})} \leq C\|\tilde{P}(t, \cdot)\|_{L^{2}(\mathcal{B})}, \\
& \|\nabla P\|_{L^{\infty}(\mathcal{B})} \leq C .
\end{aligned}
$$

Then we obtain

$$
\frac{1}{2} \frac{d}{d t}\|\tilde{P}\|_{L^{2}(\mathcal{B})}^{2} \leq 6 C\|\tilde{P}\|_{L^{2}(\mathcal{B})}^{2}-\lambda \int_{\mathcal{B}} d f(z, P)(\tilde{P}) \tilde{P} d x
$$


If Inequality (16) holds, then we infer

$$
\frac{1}{2} \frac{d}{d t}\|\tilde{P}\|_{L^{2}(\mathcal{B})}^{2} \leq(6 C-\lambda \alpha)\|\tilde{P}\|_{L^{2}(\mathcal{B})}^{2}+\lambda \beta,
$$

from which we infer $(17)$, for $c=6 C$. For $\beta=0$, the exponential decay of $\|\tilde{P}\|_{L^{2}(\mathcal{B})}$ is obvious.

Otherwise, if Inequality (18) holds, then thanks to Hölder inequality we infer

$$
\frac{1}{2} \frac{d}{d t}\|\tilde{P}\|_{L^{2}(\mathcal{B})}^{2} \leq\|\tilde{P}\|_{L^{p}(\mathcal{B})}\left(6 C\|\tilde{P}\|_{L^{q}(\mathcal{B})}-\lambda \alpha\right), \quad \text { with } q \text { such that } \quad 1 / p+1 / q=1
$$

Since $\|\tilde{P}\|_{L^{\infty}(\mathcal{B})} \leq 1$ we infer the linear decay of $\|\tilde{P}\|_{L^{2}(\mathcal{B})}^{2}$ for any $\lambda$ such that

$$
\lambda>\lambda_{0, p}:=6 C|\mathcal{B}|^{1 / q} / \alpha
$$

If $p=2,\|\tilde{P}\|_{L^{2}(\mathcal{B})}$ even decreases linearly, by obviously injecting

$$
\frac{d}{d t}\|\tilde{P}\|_{L^{2}(\mathcal{B})}^{2}=2\|\tilde{P}\|_{L^{2}(\mathcal{B})} \frac{d}{d t}\|\tilde{P}\|_{L^{2}(\mathcal{B})}
$$

in $(23)$.

\section{Joint state and parameter observer in practice}

\subsection{State observer}

We recall that in this work, we consider as measurements (denoted by $z$ ) a timecontinuous sequence of tumor masks, namely, maps taking essentially two different values inside and outside the tumor region, up to some perturbations and regularization across the front. We define $P_{\text {th }}$ as the threshold constant allowing to compare $\hat{P}$ to $z$, i.e. the region denoted by $\Omega_{\hat{P}(t)}$ and defined as $\Omega_{\hat{P}}(t)=\left\{x, \hat{P}(t, x)>P_{\text {th }}\right\}$ corresponds to the mask of the tumor at time $t$. Similarly to [9], the following state observer is proposed

$$
\left\{\begin{aligned}
\partial_{t} \hat{P}+\nabla \cdot(\hat{v} \hat{P}) & =\mathcal{L}^{0}(t, \hat{P}) \hat{P}-\lambda \delta_{\Gamma_{\hat{P}}}|\nabla \hat{P}|\left(\left(z-C_{\text {max }}^{\hat{P}}(z)\right)^{2}-\left(z-C_{\text {min }}^{\hat{P}}(z)\right)^{2}\right) \\
\hat{P}(0, \cdot) & =P_{\bullet} \\
\nabla \cdot \hat{v} & =\mathcal{L}^{0}(t, \hat{P}) \hat{P} \\
\hat{v} & =-\nabla \hat{\pi} \\
\left.\hat{\pi}\right|_{\partial \mathcal{B}} & =0
\end{aligned}\right.
$$

where $\delta_{\Gamma_{\hat{P}}}$ is the Dirac delta function of the multidimensional variable $x$ and this distribution is non-zero only on the interface $\Gamma_{\hat{P}}(t)=\left\{x, \hat{P}(t, x)=P_{\text {th }}\right\}$. The constants $C_{\max }^{\hat{P}}$ and $C_{\min }^{\hat{P}}$ are defined by $C_{\max }^{\hat{P}}=\max \left(C_{\text {in }}^{\hat{P}}, C_{\text {out }}^{\hat{P}}\right)$ and $C_{\text {min }}^{\hat{P}}=\min \left(C_{\text {in }}^{\hat{P}}, C_{\text {out }}^{\hat{P}}\right)$ with

$$
C_{\text {in }}^{\hat{P}}(z)=\frac{1}{\left|\Omega_{\hat{P}}\right|} \int_{\Omega_{\hat{P}}} z \mathrm{~d} \mathcal{B}, \quad C_{\text {out }}^{\hat{P}}(z)=\frac{1}{\left|\mathcal{B} \backslash \Omega_{\hat{P}}\right|} \int_{\mathcal{B} \backslash \Omega_{\hat{P}}} z \mathrm{~d} \mathcal{B} .
$$

The strictly positive constant $\lambda$ - called the parameter gain - balances the impact of the data-correction on the model depending on the level of confidence in data. This justifies the nudging terminology used in the data assimilation community [22]. The gain is therefore typically adjusted by considering the level of noise in data: the noisier the data, the less the value of $\lambda$ has to be. Sometimes an excessively large gain can be counter-productive (by adding noise and/or numerical instabilities) and in any case should eventually be optimized - see [34, 32, 35]. Using the formalism of Proposition 4, we have

$$
f(z, \hat{P})=-\delta_{\Gamma_{\hat{P}}}|\nabla \hat{P}|\left(\left(z-C_{\max }^{\hat{P}}(z)\right)^{2}-\left(z-C_{\min }^{\hat{P}}(z)\right)^{2}\right) .
$$


It is easy to see that $f$ satisfies Hypothesis 2 since we assume that $z=\left(C_{\min }^{P}+C_{\max }^{P}\right) / 2$ on the time-evolving tumor boundary $\Gamma_{P}$ of the target solution. The well-posedness of the observer model is not considered here due to the complexity induced by the observer correction but we assume that there exists a smooth enough solution. Our objective here is to consider the linearized version of this equation and to assess the stabilization effect of the correction term.

Remark 4. By applying Proposition 3, we directly have $0 \leq \hat{P} \leq 1$.

To analyze the observer model (25) in [9], the authors defined $h_{z}$ which can be seen as the wavelength of the front width in data as

$$
\frac{1}{h_{z}}=\min _{\partial \Omega} \frac{\partial_{n} z}{C_{\min }^{P}(z)-C_{\max }^{P}(z)}
$$

Here we assume that there $\operatorname{exists}^{1} C>0$ such that

$$
\frac{1}{h_{z}} \geq C+\frac{1}{4}\left(\frac{1}{\left|\Omega_{P}\right|}+\frac{1}{\left|\mathcal{B} \backslash \Omega_{P}\right|}\right)\left(\int_{\Gamma_{P}}|\nabla P|^{2}\right)^{\frac{1}{2}}\left(\int_{\Gamma_{P}} \frac{1}{|\nabla P|^{2}}\right)^{\frac{1}{2}} .
$$

Remark 5. Condition (28) pertains to the sharpness of the front of $z$. In other words, verifying this condition means that the image is sufficiently contrasted.

Proposition 5. Assume that:

- data $z$ satisfies (28) (i.e. $z$ is sharp enough),

- there exists $\varepsilon>0$ to be assumed small such that at any time the solution $\hat{P}$ of (25) is such that $\tilde{P}=P-\hat{P}$ has a compact support included in a tubular neighborhood of $\Gamma_{P}(t)=\left\{x, P(t, x)=P_{t h}\right\}$ of width $\varepsilon$.

Then for any $\lambda>2 c \varepsilon / \alpha$, for any $t>0$

$$
\|\tilde{P}(t)\|_{L^{2}(\mathcal{B})}^{2} \leq\|\tilde{P}(0)\|_{L^{2}(\mathcal{B})}^{2} e^{-\left(\frac{\lambda \alpha}{\varepsilon}-c\right) t}+4 \frac{\varepsilon^{2} \bar{C}}{\alpha} .
$$

Remark 6. The second hypothesis of the above Proposition is similar to assuming that the error is localized to the tumor front. This assumption is coherent in our application context, where data consists mainly of tumor segmentation.

Remark 7. The above proposition shows that the observer decreases the error exponentially fast from $\varepsilon$ to $\varepsilon^{2}$ in $L^{2}$ norm. Since the observer term only acts on the front of the tumor, one cannot show the exponential decay over time, unlike Proposition 4.

Proof. Following Proposition 4, we show the following estimates: there exists $\alpha>0$, such that

$$
\int_{\mathcal{B}} d f(z, P)(\tilde{P}) \tilde{P} \mathrm{~d} \mathcal{B} \geq \frac{\alpha}{\varepsilon}\|\tilde{P}\|_{L^{2}(\mathcal{B})}^{2}+o(\varepsilon),
$$

The proof of the last inequality is inspired from [9], where the authors have computed the following integral using Fréchet derivative formalism. For all $u \in L^{\infty}\left(0,+\infty ; H^{s}\right)$, we have

$$
\begin{aligned}
\int_{\mathcal{B}} d f(z, P)(u) u \mathrm{~d} \mathcal{B}=2\left(C_{\min }^{P}(z)-C_{\max }^{P}(z)\right) \int_{\Gamma_{P}} \partial_{n} z u^{2} \mathrm{~d} \Gamma_{P} \\
-\frac{\left(C_{\min }^{P}(z)-C_{\max }^{P}(z)\right)^{2}}{2}\left(\frac{1}{\left|\Omega_{P}\right|}+\frac{1}{\left|\mathcal{B} \backslash \Omega_{P}\right|}\right)\left(\int_{\Gamma_{P}} \frac{u}{|\nabla P|} \mathrm{d} \Gamma_{P}\right)\left(\int_{\Gamma_{P}}|\nabla P| u \mathrm{~d} \Gamma_{P}\right) .
\end{aligned}
$$

As $z$ is defined as a regularized mask of the target solution, we have $\partial_{n} z<0$ on the front of $P$. Using Cauchy Schwarz inequality, we have

$$
\int_{\Gamma_{P}} \frac{u}{|\nabla P|} \leq\left(\int_{\Gamma_{P}} u^{2}\right)^{\frac{1}{2}}\left(\int_{\Gamma_{P}} \frac{1}{|\nabla P|^{2}}\right)^{\frac{1}{2}},
$$

\footnotetext{
${ }^{1}$ For the parabolic system studied in [9], $C$ was set to zero.
} 
and

$$
\int_{\Gamma_{P}}|\nabla P| u \leq\left(\int_{\Gamma_{P}} u^{2}\right)^{\frac{1}{2}}\left(\int_{\Gamma_{P}}|\nabla P|^{2}\right)^{\frac{1}{2}},
$$

and using Inequality (28), one can prove that, for $\alpha=C \frac{\left(C_{\min }^{P}-C_{\max }^{P}\right)^{2}}{2}$,

$$
\int_{\mathcal{B}} d f(z, P)(u) u \mathrm{~d} \mathcal{B} \geq \alpha\left(\int_{\Gamma_{P}} u^{2} \mathrm{~d} \Gamma_{P}\right) .
$$

Denote by $\omega^{\varepsilon}(t)$ the support of $\tilde{P}(t, \cdot)$, which is included in the tubular neighborhood of width $\varepsilon$ of $\Gamma_{P}(t)$. Obviously,

$$
\omega^{\varepsilon}(t) \subset\left\{\Phi\left(t, x_{T}, \eta\right)=\Psi\left(t, x_{T}\right)+\eta \vec{n}\left(t, x_{T}\right) \mid\left(x_{T}, \eta\right) \in \Gamma_{P}(t) \times(-\varepsilon / 2, \varepsilon / 2)\right\},
$$

where $\left(t, x_{T}\right) \mapsto \Psi\left(t, x_{T}\right)$ is an atlas of local coordinates of $\Gamma_{P}(t), \vec{n}\left(t, x_{T}\right)$ is the outwardly directed normal vector and $\eta$ is the normal variable. This implies that

$$
\|\tilde{P}\|_{L^{2}(\mathcal{B})}^{2} 2=\int_{-\varepsilon / 2}^{\varepsilon / 2} \int_{\Gamma_{P}}(\tilde{P} \circ \Phi)^{2} \mathrm{~d} x_{T} \mathrm{~d} \eta .
$$

Using the Taylor expansion of $\tilde{P}$-assumed to be smooth enough- in the normal direction we infer

$$
\tilde{P}=\tilde{P}_{\Gamma_{P}}+\eta \partial_{n} \tilde{P}+o(\eta),
$$

and then

$$
\|\tilde{P}\|_{L^{2}(\mathcal{B})}^{2}=\varepsilon|\tilde{P}|_{L^{2}\left(\Gamma_{P}\right)}^{2}+o\left(\varepsilon^{2}\left\|\tilde{P}_{\mid t=0}\right\|_{H^{s}}\right) .
$$

We then obtain

$$
\int_{\mathcal{B}} d f(z, P)(\tilde{P}) \tilde{P} \mathrm{~d} \mathcal{B} \geq \frac{\alpha}{\varepsilon}\|\tilde{P}\|_{L^{2}(\mathcal{B})}^{2}+o\left(\varepsilon\left\|\tilde{P}_{\left.\right|_{t=0}}\right\|_{H^{s}}\right) .
$$

There exists $\bar{C}>0$, depending on $\left\|\tilde{P}_{t=0}\right\|_{H^{s}(\mathcal{B})}$ such that

$$
\int_{\mathcal{B}} d f(z, P)(\tilde{P}) \tilde{P} \mathrm{~d} \mathcal{B} \geq \frac{\alpha}{\varepsilon}\|\tilde{P}\|_{L^{2}(\mathcal{B})}^{2}-\bar{C} \varepsilon
$$

Using Proposition 4, we have:

$$
\|\tilde{P}(t)\|_{L^{2}(\mathcal{B})}^{2} \leq\|\tilde{P}(0)\|_{L^{2}(\mathcal{B})}^{2} e^{-\left(\frac{\lambda \alpha}{\varepsilon}-c\right) t}+\frac{\varepsilon^{2} \bar{C}\left(1-e^{-\left(\frac{\lambda \alpha}{\varepsilon}-c\right) t}\right)}{\alpha-\frac{c \varepsilon}{\lambda}} .
$$

Then for any $\lambda \geq 2 c \varepsilon / \alpha$, we infer the result:

$$
\|\tilde{P}(t)\|_{L^{2}(\mathcal{B})}^{2} \leq\|\tilde{P}(0)\|_{L^{2}(\mathcal{B})}^{2} e^{-\left(\frac{\lambda \alpha}{\varepsilon}-c\right) t}+4 \frac{\varepsilon^{2} \bar{C}}{\alpha} .
$$

Regarding the choice of the gain parameter, the value of $\lambda$ given in (24) is probably not optimal and only roughly evaluable in practice. In [21], a practical study to choose $\lambda$ is proposed. The objective is to choose a value of $\lambda$ not too large in order to have a good balance between model terms and the observer term and not too small to correct reasonable initial condition error. More precisely, numerical tests have shown that a value of $\lambda$ around $30 \% \frac{m_{0} V_{0}}{\max (f)}$ is a good a priori value.

Remark 8. From the above state observer one can extract the following discrepancy functional

$$
\mathcal{D}(z, \hat{P})=H\left(\hat{P}>P_{t h}\right)\left(z-C_{\max }(z, \hat{P})\right)+\left(1-H\left(\hat{P}>P_{t h}\right)\right)\left(z-C_{\text {min }}(z, \hat{P})\right),
$$

where $H$ is the Heaviside function. However, the correction term cannot be exactly rewritten as $\mathcal{G}_{y}(\mathcal{D}(z, \hat{y}))$ as presented in Equation (5) due to the fact that Heaviside distribution cannot be multiplied univocally [14]. 


\subsection{Joint state and parameter observer}

We can now present the additional level of parameter identification compatible with our state estimator. To that purpose, we follow the strategy proposed in [29] and further developed in [28]. Essentially, this assumes that an effective state observer is already available, as in our case. The functional used for the Kalman filter is adapted from the discrepancy given in Remark 8 in order to obtain a least square functional (as the disturbances of Kalman filters are assumed to be Gaussian, a least square functional is necessary) following the strategy given in [31]. For the gain $\mathcal{G}_{\theta}$ given in the parameters dynamics, see Equation (4), a reduced Kalman filter to the parameters can be used. The state gain $\mathcal{G}_{y}$ is modified in order to take into account of the reduction of the Kalman-based filter to the parameter space. Similarly to [9], we choose to work in $L^{2}$ space with its usual norm. If the error due to the initial condition is not too large it is possible to prove for linear cases that

$$
\|\hat{y}(t, \cdot)-y(t, \cdot)\|_{L^{2}(\mathcal{B})} \longrightarrow_{t \rightarrow \infty} 0, \quad \text { and } \quad \theta(t) \longrightarrow_{t \rightarrow \infty} \theta_{\diamond},
$$

see [29]. It is important to include in $\hat{y}$ all the state variables even if the state observer acts only on $\hat{P}$, see $[6]$ for more details. In our case, we have

$$
\hat{y}=\left(\hat{P}, \hat{M}, \hat{\pi}, \hat{v}, \int_{0}^{t} \hat{P}(s, x) d s\right) .
$$

A convenient Reduced-order Unscented Kalman Filter (RoUKF) is considered for our non linear model. The main advantage of RoUKF is that it does not require differentiating the model and the discrepancy with respect to the trajectory, but instead relies on "particles" surrounding the trajectory in order to compute the system sensitivities to data and uncertainties. Different choices of sigma-points can be used in practice. If we denote by $p$ be the number of estimated parameters, we consider the following $2 p$ canonical sigma points

$$
I^{(i)}= \begin{cases}\sqrt{p} e_{i}, & \text { for } 1 \leqslant i \leqslant p \\ -\sqrt{p} e_{2 p-i}, & \text { for } 0 \leqslant i \leqslant p-1,\end{cases}
$$

and the associated weights $\alpha_{i}=\frac{1}{2 p}$ are chosen all equal since we assume that every point is located on a regular polyhedron around the mean. We denote by $W_{\alpha}$ the diagonal matrix $W_{\alpha}=\operatorname{diag}\left(\alpha_{1}, \cdots, \alpha_{2 p}\right)$. It is possible to reduce the computation time by using simplex sigma points (of dimension $p+1$ ). However this is counterbalanced by the fact that the order of the parameters has an influence on the result of the computation. Actually, the triangular structure of simplex sigma points implies that the effect of one parameter interfere with the effects of the following parameters. Canonical sigma points do not present this drawback as the behaviour of each parameter is independent. We refer the interested reader to [21] for more details.

In what follows, we denote by $X^{*}$ the matrix obtained by concatenating the column vectors $X^{(i)}$ side by side. To present the RoUKF, we consider a discrete-time finite dimensional nonlinear dynamical state observer system

$$
\hat{y}_{n+1}=M_{n+1 \mid n}\left(\hat{y}_{n}, \hat{\theta}_{n}\right)+G_{n+1 \mid n}\left(z_{n}, \hat{y}_{n}\right),
$$

where $M_{n+1 \mid n}$ and $G_{n+1 \mid n}$ are the transition model and state correction operators. We introduce the 3 following matrices

$$
L_{0}^{y}=0, L_{0}^{\theta}=\mathbb{1} \text { and } U_{0}=\operatorname{Cov}\left(\xi_{\theta}\right)^{-1},
$$

which can be interpreted as the initial state and parameter sensitivities and the inverse of the initial reduced parametric covariance respectively. We denote by $R$ the inverse of the metric on the observation space $\mathcal{Z}$ corresponding to the observation covariance. This implies that similarly to $\lambda, R^{-1}$ balances the impact of the data-correction on the model depending on the level of confidence in data: the noisier the data, the less the value of $R^{-1}$ has to be.

At each time step, one iteration of the model is simulated for every particle, and the discrepancy of these particles is calculated in order to evaluate how the system of parameters needs to evolve. The complete algorithm is the following: 
Algorithm 1. Reduced-order Unscented Kalman Filter (RoUKF)

1. Sampling

$$
\left(\begin{array}{c}
\hat{y}_{n}^{(i)+} \\
\hat{\theta}_{n}^{(i)+}
\end{array}\right)=\left(\begin{array}{c}
\hat{y}_{n}^{+} \\
\hat{\theta}_{n}^{+}
\end{array}\right)+\left(\begin{array}{c}
L_{n}^{X} \\
L_{n}^{\hat{\theta}}
\end{array}\right) \sqrt{U_{n}^{-T}} I^{(i)}
$$

2. Prediction (model)

$$
\hat{y}_{n+1}^{(i)-}=M_{n+1 \mid n}\left(\hat{y}_{n}^{(i)+}, \hat{\theta}_{n}^{(i)+}\right)
$$

3. State correction (state observer)

$$
\left\{\begin{array}{l}
\hat{y}_{n+1}^{(i)+-}=\hat{y}_{n+1}^{(i)-}+G_{n+1 \mid n}\left(z_{n+1}, \hat{y}_{n+1}^{(i)-}\right) \\
\hat{y}_{n+1}^{+-}=\sum_{i=1}^{p} \alpha_{i} \hat{y}_{n+1}^{(i)+} \\
\hat{\theta}_{n+1}^{+-}=\sum_{i=1}^{p} \alpha_{i} \hat{\theta}_{n}^{(i)}
\end{array}\right.
$$

4. Parameter correction

$$
\left\{\begin{array}{l}
L_{n+1}^{X}=\hat{y}_{n+1}^{*+-} W_{\alpha} I^{*} \\
L_{n+1}^{\hat{\theta}}=\hat{\theta}_{n+1}^{*+-} W_{\alpha} I^{*} \\
\Gamma_{n+1}^{(i)}=\mathcal{D}\left(z_{n+1}, \hat{y}_{n+1}^{(i)+-}\right) \\
L_{n+1}^{\Gamma}=\Gamma_{n+1}^{*} W_{\alpha} I^{*} \\
U_{n+1}=\mathbb{1}^{+}\left(L_{n+1}^{\Gamma}\right)^{T} R^{-1} L_{n+1}^{\Gamma} \\
\hat{y}_{n+1}^{+}=\hat{y}_{n+1}^{+-}-L_{n+1}^{X} U_{n+1}^{-1}\left(L_{n+1}^{\Gamma}\right)^{T} R^{-1}\left(\sum_{i=1}^{p} \alpha_{i} \Gamma_{n+1}^{(i)}\right) \\
\hat{\theta}_{n+1}^{+}=\hat{\theta}_{n+1}^{+-}-L_{n+1}^{\hat{\theta}} U_{n+1}^{-1}\left(L_{n+1}^{\Gamma}\right)^{T} R^{-1}\left(\sum_{i=1}^{p} \alpha_{i} \Gamma_{n+1}^{(i)}\right) .
\end{array}\right.
$$

\section{Numerical validation. Illustration with synthetic data.}

\subsection{Tumor growth model}

All the numerical tests have been performed using the following tumor growth model:

$$
\left\{\begin{aligned}
\partial_{t} P+\nabla \cdot(v P) & =M P, & \mathcal{B} \\
\partial_{t} M & =-\alpha M P, & \mathcal{B} \\
\nabla \cdot v & =M P, & \mathcal{B} \\
v & =-\nabla \pi, & \mathcal{B} \\
\left.\pi\right|_{\partial \mathcal{B}} & =0, & \partial \mathcal{B} \\
P(0, \cdot) & =P \bullet(\cdot), & \mathcal{B} \\
M(0, \cdot) & =M_{0}(\cdot), & \mathcal{B}
\end{aligned}\right.
$$

where $\alpha^{-1}$ corresponds to the characteristic time at which the tumor growth carrying capacity $[12,15,3] M$ decreases and $M_{0}$ is the initial growth rate (which can be spatially distributed). Using notations of Section 3, this model corresponds to consider

$$
\mathcal{G}^{0}(t, P)=\mathcal{G}^{1}(t, P)=\mathcal{L}^{0}(t, P) P=M_{0}(\cdot) P(t, \cdot) e^{-\alpha \int_{0}^{t} P(s, \cdot) d s} .
$$

It is easy to see that $\mathcal{L}^{0}(t, \cdot)$ verifies Hypothesis 1 then using Proposition 1 , there exists a unique solution $P \in L^{\infty}\left(0, T ; H^{s}(\mathcal{B})\right)$ of System (29).

The model is implemented in $\mathrm{C}++$ by using the Cadmos library, developed in the team Monc (Inria Bordeaux Sud-Ouest), which contains solvers of advection and diffusion partial differential equations. These solvers use finite volume methods. We use a cartesian grid, which is well adapted to medical imaging. The choice of the degrees of freedom depends on the quality of the clinical image, on the computation time and on the desired numerical precision. Regarding the discretization of the state observer term (27), the main difficulty lies in the discretization of the Dirac term. This question is well-addressed in the literature and we have used the discretization proposed in [13]. More details about the numerical approximation are given in [21]. 


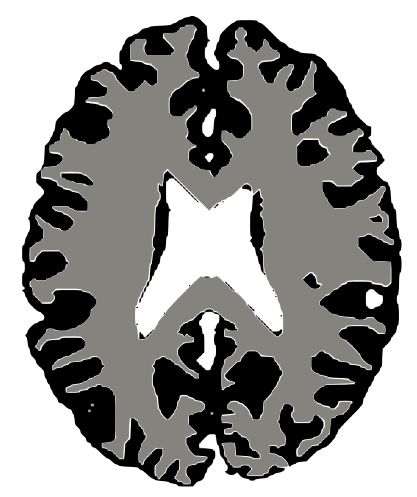

Figure 1: Computational domain for $2 \mathrm{D}$ synthetic brain tumor discretized by a grid of $181 \times 217$ points. The colors corresponds to the segmentation of grey matter (in black) and white matter (in grey). Brain ventricles are at the center of the image.
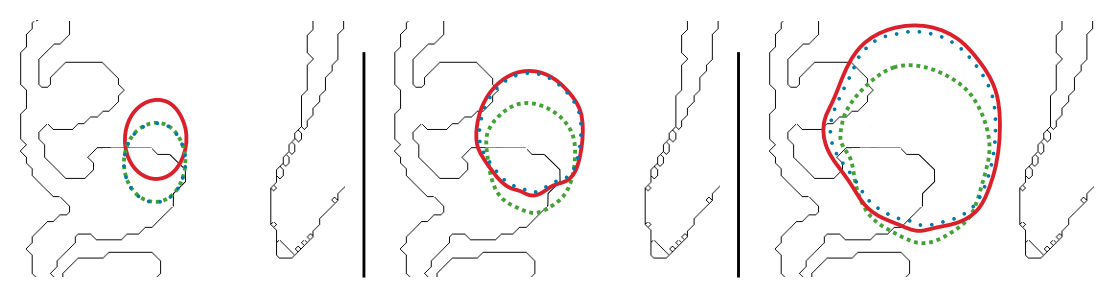

Figure 2: Comparison of tumor shapes: target (straight red line), without correction (dashed green line, $\lambda=0$ ) and with state correction (dotted blue line, $\lambda=0.03$ ). State correction is only applied between $t_{0}$ and $t_{1}$. Initial condition $\hat{P}_{0}$ is shifted from initial condition $P_{\bullet}$. The set of parameters is the same for all three cases: $\left(\alpha, m_{0}, m_{1}\right)=(0.02,0.1,0.03)$, with $m_{0}$ and $m_{1}$ the values of $M_{0}$ in white and grey matter respectively.

\subsection{Standalone state estimation}

In this part, we assume that the uncertainties are reduced to the initial condition $P_{\bullet}$ in order to numerically validate the state observer presented in Section 4.1 on $2 \mathrm{D}$ synthetic brain tumor. Figure 1 corresponds to the computational domain (discretized by a grid of $181 \times 217$ points). Segmentations of white and grey matter are presented for illustration.

On Figure 2, observations are shown in red at three times $t_{0}=0, t_{1}=20$ and $t_{2}=$ 40. State correction is effective from $t_{0}$ to $t_{1}$ and the last time $t_{2}$ is used to compare the observation and the simulated front. Initial vascularization $M_{0}$ takes different values in white and grey matters of the brain, because tumor propagation is faster in white matter than in grey matter $[33,16]$.

As we want to validate the state observer, the same set of parameters is then used for all simulations: $\left(\alpha, m_{0}, m_{1}\right)=(0.02,0.1,0.03)$, where $m_{0}$ is the value of $M_{0}$ in white matter, bigger than $m_{1}$, the value in grey matter. According to the simulation of the target solution given in Figure 2 (see the red contour evolution), we can see that the tumor zone located in grey matter grows slower than the zone located in white matter. In order to validate the state correction, the initial condition $P_{\bullet}$ of the observer model is slightly shifted from the one of the target model. This new initial condition is represented in green at time $t_{0}$ (Figure 2, left). The green contour evolution corresponds to the solution of the model when no correction is applied $(\lambda=0)$. As the tumor is initially shifted in grey matter, its growth is slower than the target solution, and we notice that the volume and the shape stay far from the target solution at $t_{1}$ and $t_{2}$ (Figure 2, middle and right). The blue contour evolution corresponds to the solution of the observer model when the state correction $(\lambda=0.03)$ is applied between $t_{0}$ and $t_{1}$, with the same shifted initial condition. On the figure, we notice 
that at time $t_{1}$, the blue and red fronts are superposed. When the correction is released, the observer front (in blue) stays close to the target front (in red), as it can be seen at time $t_{2}$. The small error observed at time $t_{2}$ comes from the fact that the extra-cellular matrix $M$ is not consumed in the same way in both cases, due to the difference of initial condition.

\subsection{State and parameters estimation. Extension to clinical cases.}

The objective of this section is to use synthetic data in order to propose the best strategy to apply to real data. In particular, the following three main difficulties are investigated here:

- Data needs to be time-interpolated because only few times are available (Section 5.3.1).

- There is a correlation between $\alpha$ and $M_{0}$ (Section 5.3.2).

- In many cases, the shape of $M_{0}$ is not known (Section 5.3.3).

To overcome the two first challenges, we introduce a volume calibration. Let the initial condition of $P$ equal to $P(0, x)=1$ inside the tumor and 0 elsewhere, one can easily see that $P(t, X)=1$ inside the tumor and 0 elsewhere at any time. We define $\Omega(t)=\{x, P(t, x)>0\}$ as the domain occupied by the tumor at time $t$ and we assume that $M_{0}$ given by (29g) is a constant field equals to a value $m_{0}$. By integrating System (29) on $\Omega(t)$ and by using Reynolds and Green theorems, one can show (see [10]) that the tumor volume defined by

$$
V(t)=\int_{\mathcal{B}} P(t, x) d x=\int_{\Omega(t)} d x \text { equals to } V(t)=V_{0} e^{\frac{m_{0}}{\alpha}\left(1-e^{-\alpha t}\right)} .
$$

This formula can be used to estimate $m_{0}$ and $\alpha$ using only the volumes of the tumor shape. This strategy has been validated with a cohort of meningiomas, see [10]. However, the parameters $\alpha$ and $m_{0}$ are correlated and non identifiable but the estimation of the volume of the tumor is quite good with only two times measurements (and excellent with three timepoints). Unfortunately, this strategy does not work for complex tumors when $M_{0}$ is a spatial field which is the main motivation of this work.

\subsubsection{Data interpolation}

In practical situations, data is not a time-continuous sequence of images, but a sequence of 2 or 3 images acquired at distant times. In order to calculate the correction terms at a given time $t$, the idea is to interpolate data. The easiest way is to use a linear interpolation. However, when the tumor volume satisfies a solvable ODE, it is more accurate to use it to interpolate data. Let us suppose that we want to interpolate data $z(t)$ with $z\left(t_{0}\right)=z_{0}$ and $z\left(t_{1}\right)=z_{1}$. First step is redistancing $z_{0}$ and $z_{1}$ which gives $\Phi_{0}$ and $\Phi_{1}$. Then we calculate

$$
\Phi(t)=\frac{V\left(t_{1}\right)-V(t)}{V\left(t_{1}\right)-V\left(t_{0}\right)} \Phi_{0}+\frac{V(t)-V\left(t_{0}\right)}{V\left(t_{1}\right)-V\left(t_{0}\right)} \Phi_{1} .
$$

We thus define $z$ between $t_{0}$ and $t_{1}$ as

$$
z(t)= \begin{cases}1 & \text { if } \Phi(t)>0 \\ 0 & \text { if } \Phi(t)<0 \\ \frac{1}{2} & \text { if } \Phi(t)=0\end{cases}
$$

\subsubsection{Correlation between $\alpha$ and $M_{0}$}

In this section, the aim is to validate parameter correction by Kalman filter. Initial conditions are supposed to be perfectly known. As said in the introduction of Section 5.3, parameters $\alpha$ and $M_{0}$ are correlated and non identifiable. Concerning $M_{0}$, the parameters which have to be estimated are $m_{0}$ and $m_{1}$, the values of $M_{0}$ in white and grey matter. Target simulation, represented in red on Figure 3, is obtained with target parameters $\left(\alpha, m_{0}, m_{1}\right)=$ $(0.001,0.08,0.01)$. Four data are extracted at the times $t_{0}=0, t_{1}=10, t_{2}=20$ and $t_{3}=30$ 
(a)
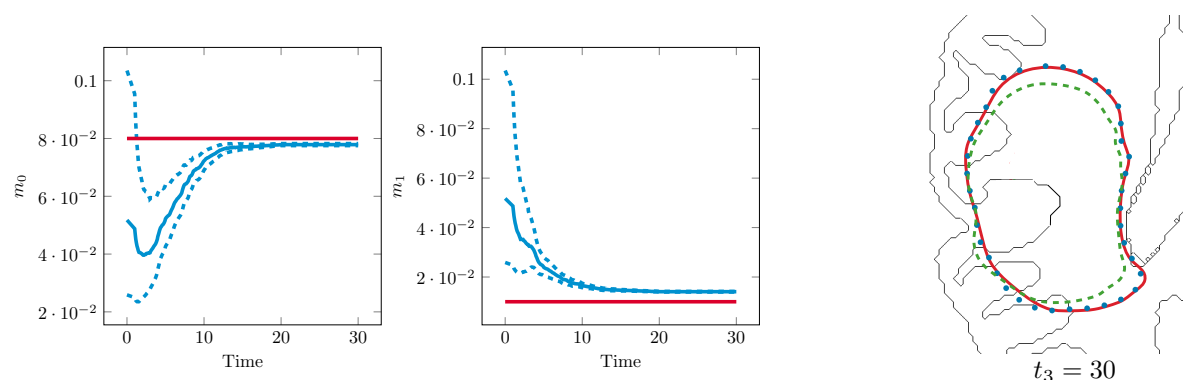

(b)
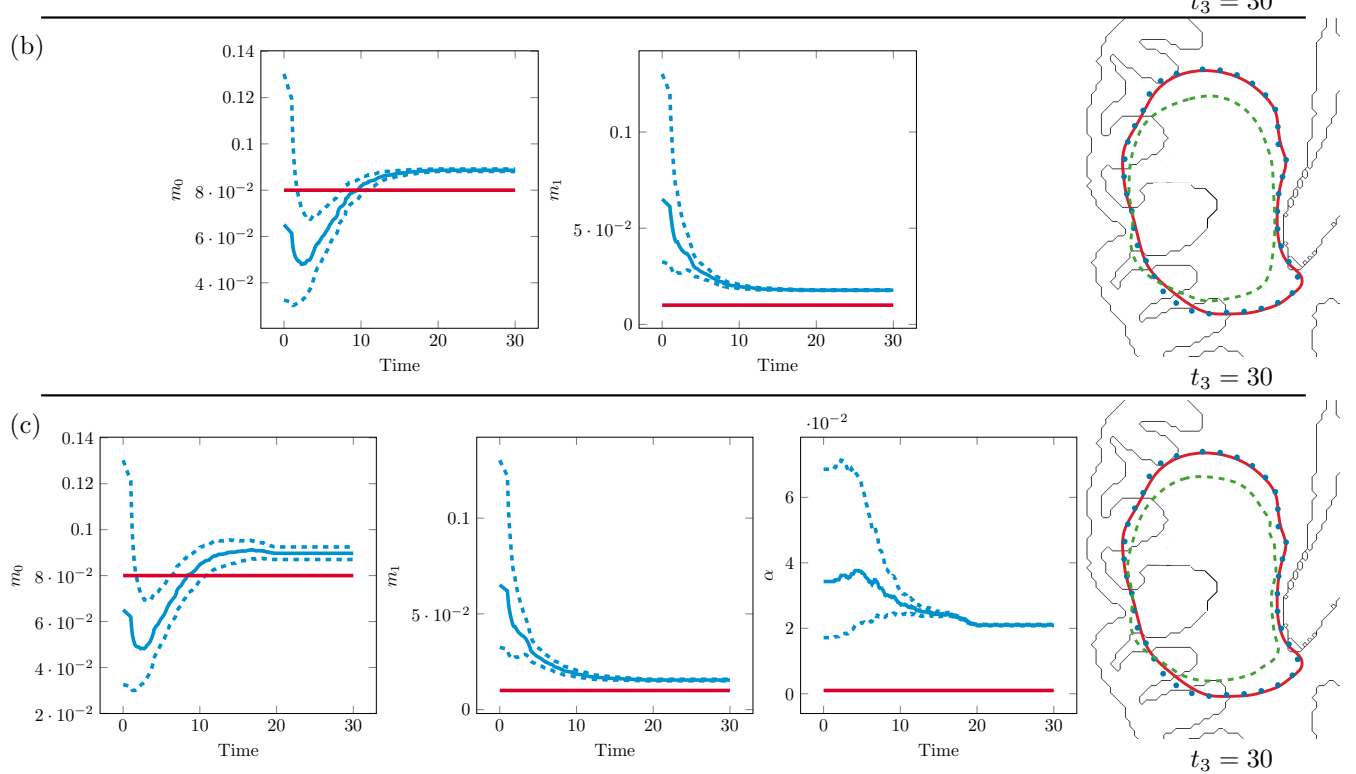

Figure 3: Illustration of joint state and parameters estimation in 3 cases: (a) $M_{0}$ is estimated and $\alpha$ is known, (b) $M_{0}$ is estimated and $\alpha$ is fixed with volume calibration, (c) $M_{0}$ and $\alpha$ are estimated. State and parameter correction is only applied between $t_{0}=0$ and $t_{2}=20$.

For each case, the curve plots show the time evolution of parameters $m_{0}, m_{1}$ and $\alpha$ (in straight blue) with standard deviation bands (in dashed blue) corresponding to Step 4 of Algorithm 1. Target values are in red. Estimated values of parameters correspond to the values at last simulation time $t_{3}=30$ (if convergence occurs).

Comparison of tumor shape at time $t_{3}=30$ are also given: target (straight red lines), without correction (dashed green lines) and with state and parameter correction (dotted blue lines, $\lambda=1 e-4)$. 


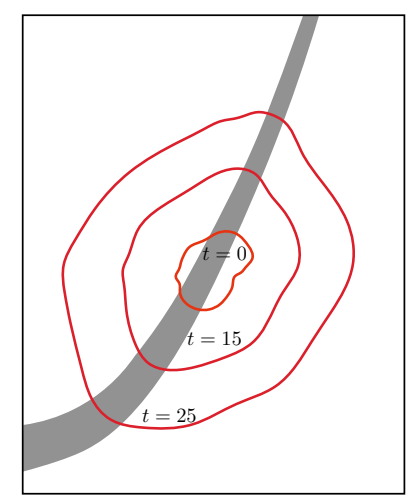

Figure 4: Synthetic data obtained at time $t_{0}=0, t_{1}=15$ and $t_{2}=25$. This simulation is obtained with $M_{0}$ taking a value $m_{\text {in }}=0.3$ inside the synthetic vessel (white front), and $m_{\text {out }}=0.08$ outside the vessel.

and only the first three tumor shapes are used for correction. The volume interpolation presented in Section 5.3.1 is applied. In the first place, we suppose that $\alpha$ is known (the same value is used for the target and the observer simulations), and we try to estimate $M_{0}$ in order to validate the model on a synthetic case. In the second place, $\alpha$ is not supposed to be known, and it is fixed with the volume calibration (using the volumes at $t_{0}, t_{1}$ and $t_{2}$ ). Finally, $\alpha$ is added to the list of parameters estimated by the Kalman filter. These three methods are tested in order to validate the method and to see which one is the best to apply to real data. Figure 3 illustrates the results. The parameters have been efficiently estimated in the first case when $\alpha$ is fixed. The estimation is acceptable when $\alpha$ is fixed with the volume calibration or estimated with the Kalman filter but not perfect due to the correlation between $\alpha$ and $M_{0}$. But if we compare the tumor shapes (see the last column of Figure 3) at time $t_{3}$ (used as a prediction time), the observer state is very effectively corrected, as the estimated state is very close to the target state for three cases which is the objective of our work. In real cases, we would fix $\alpha$ with the $0 \mathrm{D}$ estimation because it is less expensive for equivalent results when focusing of shape estimation.

\subsubsection{Estimation of $M_{0}$ on a grid}

Previous sections focus only on the difference of propagation in white and grey matter. In practical cases, fibers and vessels surrounding the tumor may also lead to shape changes. Moreover, the segmentation of white and grey matter contains uncertainties and is computationally costly. The idea here is to have no prior on the structure of $M_{0}$ and to estimate it. $M_{0}$ is then estimated on a grid. Each case of the grid corresponds to a value $m_{0}$ that has to be estimated. The chosen grid is less refined that the computation grid, as Kalman filter is a costly algorithm. In order to simulate the growth of a tumor along a vessel, a target simulation is launched with $M_{0}$ taking a higher value inside a synthetic vessel. The simulation is shown on Figure 4, with the vessel front represented in white. Let us note that the tumor expands in the direction of the vessel as expected. The parameters used for this simulation are $\left(\alpha, m_{\text {in }}, m_{\text {out }}\right)=(0.02,0.3,0.08)$, with $m_{\text {in }}$ and $m_{\text {out }}$ the values of $M_{0}$ inside and outside the vessel. Our objective is here to predict the tumor shape and to compare the real value of $M_{0}$ to the one estimated by our model. Only three times are used for the estimation process.

The volume calibration gives $\left(\alpha, m_{\text {unif }}\right)=(0.029,0.132)$. Only $M_{0}$ is estimated by the joint state and parameter observer using as a-priori the value of the volume calibration. On the top of the first column of Figure 5, the first grid considered for $M_{0}$ is represented. On the bottom, the solutions of the target model in red and of the observer model in green are compared at time $t=25$. We notice that the grid $5 \times 5$ contains three types of zones. Grey zones are those which are not reached by the tumor: parameters are not corrected, and stay 

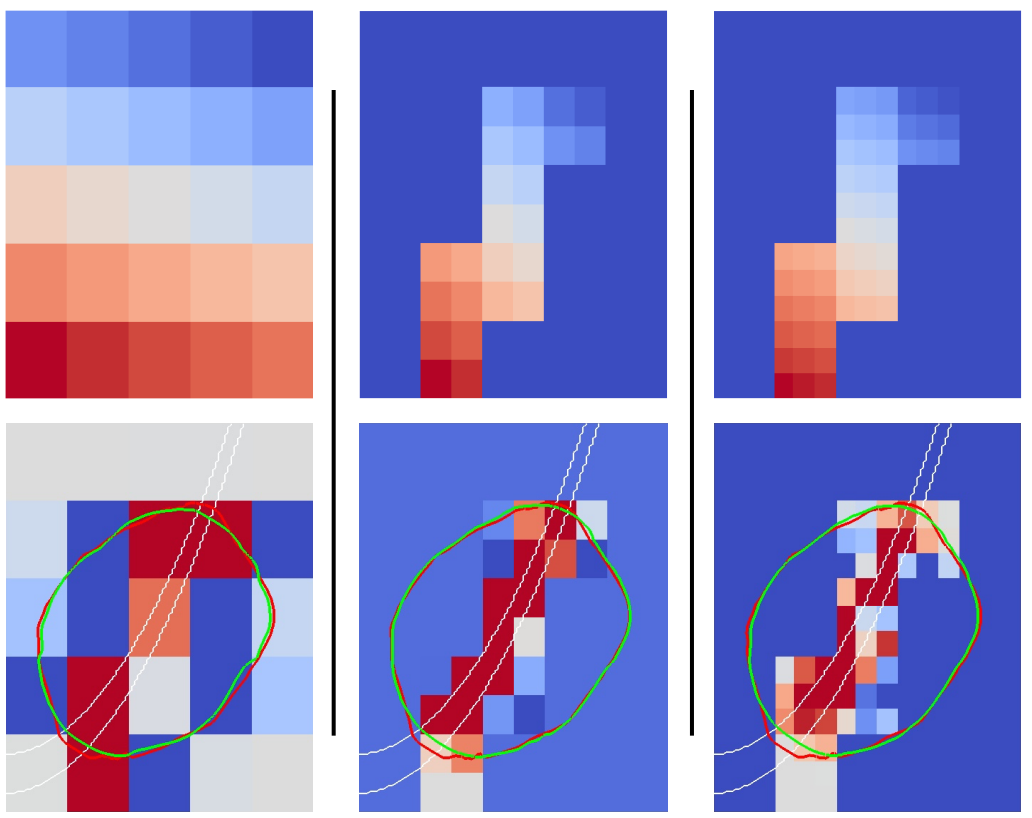

Figure 5: $M_{0}$ initial shapes (first line) and comparison between the solution of the target model and the solution of the observer model at time $t=25$ (second line). First column: initial grid. Second and third columns: evolution of the grid using the previous results.

at initial value. Red and blue zones are those where the parameter increase or decrease respectively. In these simulations, we choose $\lambda=0.001$ and an error of covariance of 0.08 . It is interesting to notice that the red zones are those located inside the vessel. It shows that it is possible to extract information on the structure of $M_{0}$, even when we have no a-priori on it. In order to improve the prediction, the grid is refined like this:

- red zones are refined,

- blue and grey zones are unified to form a single zone.

The aim is to refine interesting zones only. Unifying other zones aims at reducing the number of parameters and have reasonable computation time. We obtain successively the grids represented on the top of the second and third columns of Figure 5.

Solutions of the target and the observer models at time $t=25$ on these grids are represented on the bottom of the second and third columns of Figure 5. We notice as before that red zones are those located in the synthetic vessel. We also notice that the most refined grid is not the one that gives the best result. Each zone being correlated to its neighbours, this method cannot give a precise vascularization map. However, the method is efficient to estimate a global behaviour of the shape of $M_{0}$.

\section{Conclusion}

The objective of this paper was to propose a strategy allowing to estimate and to predict the tumor shape evolution. For this purpose, we have proposed a state observer for a hyperbolic/elliptic tumor growth model, and for data corresponding to the tumor mask over time. The state observer has been mathematically validated. Indeed, we have given sufficient conditions allowing to design a state observer and we have described a state observer verifying these conditions in a linearized situation. Then a joint state-parameter correction has been developed, as initiated in [29] by coupling the state observer with a reduced-order Kalman filter $[28,27]$ to correct the parameters. The strategy has been validated with synthetic data but in a clinical context. For this purpose, we have presented strategies to deal with the fact that data is very sparse in time and that the initial shape of the proliferation 
rate is unknown. The results on synthetic data are very promising and work is ongoing on applying this strategy in a clinical context with real data including a careful investigation of the influence of data uncertainties on the results.

\section{References}

[1] S. Alinhac and P. Gérard. Pseudo-differential operators and the Nash-Moser theorem, volume 82 of Graduate Studies in Mathematics. American Mathematical Society, Providence, RI, 2007. Translated from the 1991 French original by Stephen S. Wilson.

[2] P. Arbogast, O. Pannekoucke, L. Raynaud, R. Lalanne, and E. Mémin. Object-oriented processing of CRM precipitation forecasts by stochastic filtering. Q.J.R. Meteorol. Soc., 142(700):2827-2838, 2016.

[3] E. Baratchart, S. Benzekry, A. Bikfalvi, T. Colin, L. S. Cooley, R. Pineau, E. J. Ribot, O. Saut, and W. Souleyreau. Computational modelling of metastasis development in renal cell carcinoma. PLOS Computational Biology, 11(11):1-23, 112015.

[4] D. Bresch, T. Colin, E. Grenier, B. Ribba, and O. Saut. Computational modeling of solid tumor growth: the avascular stage. SIAM Journal on Scientific Computing, 32(4):2321-2344, 2010.

[5] H. Brezis. Functional analysis, Sobolev spaces and partial differential equations. Springer Science \& Business Media, 2010.

[6] D. Chapelle, A. Gariah, P. Moireau, and J. Sainte-Marie. A Galerkin strategy with proper orthogonal decomposition for parameter-dependent problems-Analysis, assessments and applications to parameter estimation. ESAIM: Mathematical Modelling and Numerical Analysis, 47(6):1821-1843, 2013.

[7] T. Colin, F. Cornelis, J. Jouganous, J. Palussière, and O. Saut. Patient-specific simulation of tumor growth, response to the treatment, and relapse of a lung metastasis: a clinical case. $J$ Comput Surg, 2(1):1, Dec. 2015.

[8] T. Colin, F. Cornelis, J. Jouganous, J. Palussière, and O. Saut. Patient-specific simulation of tumor growth, response to the treatment, and relapse of a lung metastasis: a clinical case. Journal of Computational Surgery, 2(1):1, Feb 2015.

[9] A. Collin, D. Chapelle, and P. Moireau. A Luenberger observer for reaction-diffusion models with front position data. Journal of Computational Physics, 300:288-307, 2015.

[10] A. Collin, C. Copol, T. Colin, H. Loiseau, O. Saut, and T. B. Spatial mechanistic modeling for prediction of the growth of asymptomatic meningioma. 2019.

[11] F. Cornelis, M. Martin, O. Saut, X. Buy, M. Kind, J. Palussiere, and T. Colin. Precision of manual two-dimensional segmentations of lung and liver metastases and its impact on tumour response assessment using RECIST 1.1. European radiology experimental, 1(1):16, 2017.

[12] H. Enderling and M. Chaplain. Mathematical modeling of tumor growth and treatment. Current pharmaceutical design, 20, 112013.

[13] B. Engquist, A.-K. Tornberg, and R. Tsai. Discretization of Dirac delta functions in level set methods. Journal of Computational Physics, 207(1):28-51, 2005.

[14] B. Fisher. The product of distributions. The Quarterly Journal of Mathematics, 1971.

[15] P. Gerlee and A. R. A. Anderson. The evolution of carrying capacity in constrained and expanding tumour cell populations. Physical Biology, 12(5):056001, aug 2015.

[16] H. L. P. Harpold, E. C. Alvord, and K. R. Swanson. The Evolution of Mathematical Modeling of Glioma Proliferation and Invasion. J Neuropathol Exp Neurol, 66(1):1-9, Jan. 2007.

[17] C. Hogea, C. Davatzikos, and G. Biros. An image-driven parameter estimation problem for a reaction-diffusion glioma growth model with mass effects. Journal of Mathematical Biology, 56(6):793-825, 2008.

[18] P. R. Jackson, J. Juliano, A. Hawkins-Daarud, R. C. Rockne, and K. R. Swanson. Patientspecific mathematical neuro-oncology: Using a simple proliferation and invasion tumor model to inform clinical practice. Bulletin of Mathematical Biology, 77(5):846-856, May 2015.

[19] J. Jacobs, R. C. Rockne, A. J. Hawkins-Daarud, P. R. Jackson, S. K. Johnston, P. Kinahan, and K. R. Swanson. Improved model prediction of glioma growth utilizing tissue-specific boundary effects. Mathematical Biosciences, 312:59 - 66, 2019. 
[20] E. Konukoglu, O. Clatz, B. H. Menze, B. Stieltjes, M.-A. Weber, E. Mandonnet, H. Delingette, and N. Ayache. Image guided personalization of reaction-diffusion type tumor growth models using modified anisotropic eikonal equations. Medical Imaging, IEEE Transactions, 29(1):7795, 2010.

[21] T. Kritter. Utilisation de données cliniques pour la construction de modèles en oncologie. PhD thesis, Bordeaux, 2018.

[22] S. Lakshmivarahan and J. Lewis. Nudging methods: A critical overview. In S. Park and L. Xu, editors, Data Assimilation for Atmospheric, Oceanic, and Hydrologic Applications, volume XVIII. Springer, 2008.

[23] G. Lefebvre, F. Cornelis, P. Cumsille, T. Colin, C. Poignard, and O. Saut. Spatial modelling of tumour drug resistance: the case of GIST liver metastases. Mathematical Medicine and Biology: A Journal of the IMA, 34(2):151-176, 032016.

[24] L. Li, F. X. L. Dimet, J. Ma, and A. Vidard. A Level-Set-Based Image Assimilation Method: Potential Applications for Predicting the Movement of Oil Spills. IEEE Transactions on Geoscience and Remote Sensing, 55(11):6330-6343, 2017.

[25] D. Luenberger. An introduction to observers. IEEE Transactions on Automatic Control, 16:596-602, 1971.

[26] M. L. Martins, S. C. Ferreira, and M. J. Vilela. Multiscale models for the growth of avascular tumors. Physics of Life Reviews, 4:128-156, June 2007.

[27] P. Moireau and D. Chapelle. Erratum of article "Reduced-order Unscented Kalman Filtering with application to parameter identification in large-dimensional systems". ESAIM: Control, Optimisation and Calculus of Variations, 17(2):406-409, 2011.

[28] P. Moireau and D. Chapelle. Reduced-order Unscented Kalman Filtering with application to parameter identification in large-dimensional systems. ESAIM: Control, Optimisation and Calculus of Variations, 17(2):380-405, 2011.

[29] P. Moireau, D. Chapelle, and P. Le Tallec. Joint state and parameter estimation for distributed mechanical systems. Computer Methods in Applied Mechanics and Engineering, 1987(6-8):659677,2008

[30] R. H. Reichle. Data assimilation methods in the Earth sciences. Advances in Water Resources, 31:1411-1418, 2008

[31] M. Rochoux, A. Collin, C. Zhang, A. Trouvé, D. Lucor, and P. Moireau. Front shape similarity measure for shape-oriented sensitivity analysis and data assimilation for Eikonal equation. ESAIM: Proceedings and Surveys, pages 1-22, 2017.

[32] D. R. Stauffer and J.-W. Bao. Optimal determination of nudging coefficients using the adjoint equations. Tellus A, 45(5):358-369, 1993.

[33] K. R. Swanson, E. C. Alvord, and J. D. Murray. A quantitative model for differential motility of gliomas in grey and white matter. Cell Prolif., 33(5):317-329, 2000.

[34] P. Vidard, F.-X. Le Dimet, and A. Piacentini. Determination of optimal nudging coefficients. Tellus A, 55(1):1-15, 2003.

[35] X. Zou, I. Navon, and F.-X. Le Dimet. An optimal nudging data assimilation scheme using parameter estimation. Quarterly Journal of the Royal Meteorological Society, 118(508):11631186, 1992. 